\title{
Impact of Same-Session Trabectome Surgery on Ahmed Glaucoma Valve Outcomes
}

Hamed Esfandiari; ${ }^{1,2}$ Tarek Shazly; ${ }^{1}$ Priyal Shah; ${ }^{1}$ Kiana Hassanpour; ${ }^{2}$ Pooya Torkian; ${ }^{2}$ Mehdi Yaseri; ${ }^{3}$ Nils A. Loewen; ${ }^{1^{*}}$

${ }^{1}$ Department of Ophthalmology, School of Medicine, University of Pittsburgh, Pittsburgh, Pennsylvania, United States

${ }^{2}$ Ophthalmic Research Center, Shahid Beheshti University of Medical Sciences, Tehran, Iran

${ }^{3}$ Department of Epidemiology and Biostatistics, School of Public Health, Tehran University of Medical Sciences, Tehran, Iran

*Correspondence to Nils Loewen, MD, PhD

203 Lothrop St, Suite 819, Pittsburgh, PA 15213

Department of Ophthalmology, School of Medicine, University of Pittsburgh, Pittsburgh, Pennsylvania, United States.

Email: loewen.nils@gmail.com 


\section{Abstract}

Purpose: To evaluate the efficacy and survival rates of same session ab interno trabeculectomy with the trabectome and Ahmed glaucoma valve implant (AT) in comparison to the Ahmed glaucoma valve alone (A).

Method: A total of 107 eyes undergoing primary glaucoma surgery were enrolled in this retrospective comparative case series, including 48 eyes which underwent AT and 59 eyes which received $A$ alone. Participants were identified using the procedural terminology codes, and their medical records were reviewed. The primary outcome measure was surgical success, defined as intraocular pressure (IOP) $>5 \mathrm{mmHg}, \leq 21$ $\mathrm{mmHg}$, and IOP reduction $\geq 20 \%$ from baseline at two consecutive visits after three months, no reoperation for glaucoma. Secondary outcome measures were IOP, the number of glaucoma medications, incidence of a hypertensive phase, and best corrected visual acuity (BCVA).

Results: The cumulative probability of success at one year was $70 \%$ in AT, and $65 \%$ in $A(p=0.85)$. IOP decreased significantly from $26.6 \pm 10.1 \mathrm{mmHg}$ at baseline to $14.7 \pm 3.3 \mathrm{mmHg}$ at the final follow-up in AT ( $p=$ $0.001)$. The corresponding numbers for $A$ were $28.8 \pm 10.2$ and $16.7 \pm 4.9$, respectively $(p=0.001)$. The final IOP was significantly lower in AT $(p=0.022)$. The number of medications at baseline was comparable in both groups ( $2.6 \pm 1.2$ in AT and $2.5 \pm 1.3$ in $A, p=0.851$ ). Corresponding number at 1 year visit was $1.2 \pm 2$ in AT and $2.8 \pm 1.8$ in $A(p=0.001)$.

The incidence of a hypertensive phase was $18.7 \%$ in AT and $35.5 \%$ in A $(p=0.05)$. HP resolved in only $30 \%$ of eyes. The criteria for HP resolution were fulfilled in 9 eyes (30\%). There was no difference in the rate of resolution of the hypertensive phase between $A T$ and $A(33.3 \%$ and $28.5 \%$, respectively, $p=0.67)$.

Conclusion: Ahmed glaucoma valve implant with same session trabectome surgery significantly decreased the rate of the hypertensive phase and postoperative IOP as well as the number of glaucoma medications.

Keywords: Ahmed glaucoma valve; trabectome surgery; hypertensive phase; glaucoma surgery 


\section{Introduction}

Trabeculectomy and glaucoma drainage devices (GDD) are common surgical interventions for advanced glaucomas [1-3]. Concerns about bleb-related complications along with studies that indicated an equal or even higher success rate of glaucoma drainage devices have contributed to their increased use [2, 4]. The shift in practice pattern from trabeculectomy to GDDs in severe glaucoma is evidenced by a $43 \%$ decrease in the number of trabeculectomies and a 184\% increase in GDDs in the United States between 1995 and 2004 [5, 6].

The two most common GDDs in the US are non-valved Baerveldt implants (BI, Advanced Medical Optics, Santa Ana, California, USA) and the valved Ahmed valve implants (AGV, New World Medical Inc, Rancho Cucamonga, California, USA) [7]. While BIs require an intraoperative flow restriction to allow capsule formation around the plate, AGVs offer an immediate intraocular pressure control (IOP) [8, 9]. Although the average IOP following AGV is about $1.85 \mathrm{mmHg}$ higher than in $\mathrm{BI}$, a comparable success rate, fewer postoperative complication rates, and ease of handling are advantages of AGV over BI [4]. A downside of AGV is an increased rate of a hypertensive phase (HP) that can persist if not managed aggressively [10] with an incidence that ranges from $30 \%$ to $82 \%$ and starts one to 13 weeks postoperatively [10-13]. It has been speculated that early contact of aqueous inflammatory mediators with Tenon's capsule and the conjunctiva or early stretch [14] of the forming capsule may lead to this $[10,15,16]$. The management strategies for HP include early initiation of aqueous suppressant treatment after AGV implantation [16], steroid injection at the time surgery [17], antimetabolites, digital massage [18], bleb needling, and surgical excision of the bleb [8]. While GDDs bypass the conventional outflow, the Trabectome surgical system (NeoMedix, Tustin, CA, USA) enhances the conventional outflow [19]. It has a long track record of safety and efficacy in different types and severity of glaucoma [20, 21].

We hypothesized that the addition of trabectome surgery not only compensates for a higher IOP in AGVs compared to $\mathrm{BI}$ but also decreases the incidence of hypertensive phase by reducing the levels of aqueous humor inflammatory mediators surrounding the plate and overlying tissue.

\section{Methods}

The protocol of this study was approved by the Institutional Review Board of the University of Pittsburgh Human Subjects Research Committee and adhered to the tenets of the Declaration of Helsinki and regulations of the Health Insurance Portability and Accountability Act.

Patients who underwent either AGV with trabectome surgery (AT) or AGV alone (A) between 2008 and 2015 were identified using Current Procedural Terminology codes. Four glaucoma fellowship-trained surgeons performed the procedures. Patients older than 20 years with medically uncontrolled IOP were included in this study. Exclusion criteria were a history of uveitic glaucoma, prior ocular surgery (except uncomplicated phacoemulsification) and neovascular glaucoma. Data were collected from patients' electronic medical record and included basic demographic information, type of glaucoma and preoperative diagnosis, preoperative IOP and the number of glaucoma medications, baseline best corrected visual acuity (BCVA), type of operation, as well as the postoperative data for IOP, number of medications, and BCVA.

The primary outcome measure was success defined as IOP $>5 \mathrm{mmHg}, \leq 21 \mathrm{mmHg}$ and $\geq 20 \%$ reduction of IOP from baseline at two consecutive visits after three months, no need for further glaucoma surgery [22]. Eyes that had not failed and were not receiving glaucoma medical therapy were considered complete successes, 
and those requiring adjunctive medical therapy were defined as qualified successes. The secondary outcome measures were IOP, the rate of a hypertensive phase, BCVA, and the number of medications.

The hypertensive phase (HP) was defined as an IOP above $21 \mathrm{mmHg}$ during the first 3 months after surgery (with or without medications) after reduction of IOP to less than $22 \mathrm{mmHg}$ was achieved within the first week postoperatively [10]. High IOP must not have been secondary to tube occlusion, malposition, or valve dysfunction. A valve dysfunction was diagnosed if the IOP did not fall immediately after the operation. The resolution of hypertensive phase was defined as an IOP $<22 \mathrm{mmHg}$ and a reduction of the IOP by $3 \mathrm{mmHg}$ with the same number of medications or less, or a reduction of at least one medication with a change of IOP $<$ $3 \mathrm{mmHg}$ [10]. In all cases, the IOP was measured with a Goldmann applanation tonometer (GAT; Haag-Streit, Konig, Switzerland) at 1 day, 1 week, $4 \pm 1$ week, 2-4 months, 5-7 months, 8-10 months, and 11-13 months. If more than one visit was found at these intervals, the visit closest to month 6, 9, or 12 was chosen.

\section{Surgical technique}

In AT, the Trabectome (NeoMedix, Tustin, CA, USA) surgery was performed first as described in the following. The patient's head was tilted away, and the microscope was tilted toward the surgeon by about $30^{\circ}$. A temporal $1.6 \mathrm{~mm}$ clear corneal incision that was iris-parallel was created, and the handpiece was advanced into the anterior chamber. Under direct gonioscopic view, the tip of the trabectome was engaged with the trabecular meshwork (TM) at the nasal angle; ablation was started and continued for $60^{\circ}$ counterclockwise followed by a $60^{\circ}$ clockwise move to opposite direction for an approximately $120^{\circ} \mathrm{TM}$ ablation. The handpiece was withdrawn from the anterior chamber, and the incision was hydrated to seal.

In AGV, a 7-0 traction suture was placed through the supratemporal perilimbal cornea. An eight millimeter long, limbus-parallel incision through the conjunctiva and Tenon's capsule, five millimeters posterior to the limbus was created in the supratemporal quadrant, and Tenon's capsule was dissected away from the sclera with Westcott scissors to provide space for the plate insertion. The device (Ahmed glaucoma drainage implant, model FP7, New World Medical, Rancho Cucamonga, California) was primed with $2 \mathrm{ml}$ of buffered saline solution (BSS) and gently pushed through the incision into the subtenon space. The plate was secured to the sclera $10 \mathrm{~mm}$ posterior to the limbus using an 8-0 nylon suture. The tube was trimmed bevel up with an estimated intracameral length of $2 \mathrm{~mm}$. A 23-gauge needle was inserted into the anterior chamber bevel up, parallel to the iris and $1 \mathrm{~mm}$ posterior to the limbus. The tube was passed through the tunnel into the anterior chamber and secured to the sclera with a 10-0 nylon suture. A $5 \times 8 \mathrm{~mm}$ scleral patch graft was placed over the tube. Tenon's capsule and the conjunctiva were closed using a running 10-0 nylon mattress suture. The postoperative regimen consisted of moxifloxacin eye drops four times daily for one week and $1 \%$ prednisolone acetate four times per day for one month followed by gradual taper.

\section{Statistical analysis}

All analyses were performed using SPSS software (SPSS Statistics for Windows, Version 22, Armonk, NY, IBM Corporation) to describe data, frequency, and percent; mean $\pm S D$, median, and range were used. To evaluate the baseline differences between two groups, we used ANOVA, Kruskal-Wallis, Chi-Square, and Fisher exact test. To compare the rate of changes between two groups during the study follow-ups, we used interaction analysis within a linear mixed model. To compare the results between the groups adjusted for the baseline values, we used an Analysis of Covariance (ANCOVA). The difference in IOP and the number of glaucoma medications between two groups were assessed by Mann-Whitney test and independent T-test. To analyze 
the survival of subjects in the groups, Kaplan-Meier survival plots were constructed and compared using the log-rank test. Statistical significance was defined as $p \leq 0.05$. Success was defined as IOP $\leq 21 \mathrm{mmHg},>5 \mathrm{mmHg}$, and $a>20 \%$ reduction from baseline with no need for further surgery.

\section{Results}

A total of 107 eyes were included in this study consisting of 48 AT and 59 A. The baseline patient demographics are presented in Table 1. The mean age of the study participants was $64.4 \pm 15.6$ years in AT and $69.3 \pm 15.1$ years in $A(0.107)$. Thirty patients $(29.4 \%)$ underwent phacoemulsification at the time of glaucoma surgery, 12 eyes (27.9\%) in AT and 18 Eyes (30.5\%) in A (0.776). Primary open-angle glaucoma was the most common diagnosis in both groups (79.2\% and $71.2 \%$ in AT and $A$, respectively, $p=0.680$ ). There were no significant differences between the study groups regarding gender, preoperative intraocular pressure, baseline number of glaucoma medications, and type of glaucoma (Table 1).

The Kaplan-Meier survival curves (Figure 1) indicated a mean duration of survival of $10.65 \pm 0.41$ months in AT group and $10.48 \pm 0.46$ in A with no statistically significant difference between two groups (LogRank=0.03, $p=$ 0.85). The cumulative probability of success at 6 months and 12 months was $77.5 \%$ and $70 \%$ in AT, and $75 \%$ and $65 \%$ in A. The complete success was $56.2 \%$ in AT and $13.5 \%$ in A. At the final follow-up visits, 27 eyes in AT and 8 eyes in $A$ did not use glaucoma medications. In the subgroup analysis, the mean survival duration was $10.61 \pm 0.7$ months in AT with phacoemulsification versus $10.4 \pm 1.23$ months in A with phacoemulsification (LogRank=0.39, $p=0.53$ ). Corresponding values for glaucoma surgery alone were $10.6 \pm 0.51$ and $10.38 \pm 0.55$ in AT and A, respectively (LogRank=0.002, $p=0.96$, Figure 1).

IOP was decreased significantly from $26.6 \pm 10.1 \mathrm{mmHg}$ at baseline to $14.7 \pm 3.3 \mathrm{mmHg}$ at final follow-up in AT $(p=0.001$, Figure 2). The corresponding numbers for $A$ were $28.8 \pm 10.2$ and $16.7 \pm 4.9$, respectively $(p=0.001$, Table 2). During the 1-year follow-up, 6 (12.5\%) patients in AT and 5 (8.4\%) patients in A group experienced hypotony, respectively $(p=0.12)$.

IOP was significantly lower in AT at the final follow-up visit $(p=0.022)$. Eyes in AT experienced an $11.49 \pm 8.8$ $\mathrm{mmHg}$ reduction in IOP one year after the surgery compared to a $12.17 \pm 10.16 \mathrm{mmHg}$ reduction in $A(p=$ 0.830 , Figure 2). IOP was comparable on day one between AT with phacoemulsification and AT (10.3 \pm 7.1 $\mathrm{mmHg}$ versus $12.1 \pm 8.7 \mathrm{mmHg}, \mathrm{p}=0.63$ ). A combined with phacoemulsification had a significantly higher IOP on day one compared to $A(18.2 \pm 9.4$ versus $13.3 \pm 8.4, p=0.004)$.

Nine eyes in AT (18.7\%) and 21 eyes in A (35.5\%) experienced a hypertensive phase within three months of operation (0.51). Logistic regression analyses were performed to explore what factors were associated with HP development. Results indicated that the preoperative IOP was significant in the multivariate outcome (Table 3). The criteria for HP resolution were fulfilled in 9 eyes (30\%). There was no difference in the rate of HP resolution between $\mathrm{AT}$ and $\mathrm{A}$ (33.3\% and $28.5 \%$, respectively, $\mathrm{p}=0.67$ ).

The baseline number of glaucoma medications was $2.6 \pm 1.2$ in AT and $2.5 \pm 1.3$ in $A(p=0.851)$. At the final follow-up visit, the number of glaucoma medications was $1.2 \pm 1.1$ drops in AT and $2.8 \pm 1.8$ in $A(p=0.001)$. While there was a significant reduction in the number of medications in AT $(p=0.01)$, glaucoma medications remained unchanged at one-year follow-up visit in $A(p=0.21)$. 
The mean BCVA at the baseline was $0.56 \pm 0.56 \log M A R$ in AT and $0.71 \pm 0.88 \log M A R$ in $A(p=0.122)$. Corresponding numbers for final follow-up visit were $0.62 \pm 1.07 \log M A R$ and $0.83 \pm 0.97 \log M A R$, respectively $(p=0.468)$.

\section{Discussion}

This study evaluated the effect of same-session Trabectome surgery on the outcomes of Ahmed glaucoma valve implantation. AT and A were both effective in reducing IOP. The IOP reduction of $40 \%$ at the 1 -year follow-up was similar to previous reports $[2,7,8,17]$. Adding trabectome surgery led to a significantly lower IOP with fewer glaucoma medications throughout the postoperative follow-up visits. Also, a same-session trabectome surgery blunted potentially dangerous post-phacoemulsification IOP spikes, consistent with our previous studies on this subject [23].

The IOP after GDD implantation has typically two phases: 7 to 10 days of relative hypotony that may be followed by the rise of intraocular pressure above $21 \mathrm{mmHg}$ during the hypertensive phase [24]. This sequence was first described for the Molteno device and was addressed by a complete intraoperative tube ligation to prevent aqueous from negatively impacting the bleb maturation [25]. More recent studies of these devices do not describe a significant hypertensive phase [9, 26-28]. Aqueous humor of patients with glaucoma contains a high concentration of tissue growth factor $b$ (TGF-b), which has a substantial regulatory effect on the extracellular matrix macromolecules and synthesis of matrix protein as well as an inhibitory effect on degradative proteolytic enzymes [15].

The incidence of a hypertensive phase in our study was $28.1 \%$, lower but not inconsistent with previous studies $[10,16,24]$. We observed significantly fewer cases of hypertension phase in the AT group with only 18.7\%. Trabectome surgery enhances the physiologic outflow by removing the trabecular meshwork and reduces the inflammatory cytokines and stretch in the AGV bleb. In another study with the same rationale as ours, Pakravan et al. randomized eyes that underwent AGV to either starting aqueous suppressants once postoperative IOP raised to $10 \mathrm{mmHg}$ or routine follow-up where glaucoma medications were commenced if deemed necessary by the surgeon [16]. The incidence of HP in the former group was $23 \%$ compared to $60 \%$ in the routine follow-up. The results of our study match this finding and corroborate the role of aqueous inflammatory mediators and capsule stretch in promoting hypertensive phase.

Another explanation for a lower incidence of HP in AT is the effect of trabectome surgery itself. Trabectome is shown to decreases IOP by $30 \%$ from the baseline and reduces the number of glaucoma medications by $38 \%$ at seven years follow-up [20, 29].

HP has been described in up to $80 \%$ after AGV, but few studies have evaluated the clinical course of this phenomenon $[10,11,24]$. Most reports on post-GDD hypertensive phase showed relative reversibility of IOP in a significant number of patients, hence the term hypertensive "phase." However, most of these studies based their conclusion on IOP reduction without considering the effect of glaucoma medications on IOP. To address this issue, we incorporated changes in both IOP and glaucoma medications in our definition of HP resolution. Applying this definition, HP resolved in $30 \%$ of the cases, which agrees with the $28 \%$ reported by Nouri-Mahdavi and Caprioli [10]. Development of high IOP in the early postoperative period is an unfavorable prognostic factor and indicates the vigilant monitoring of IOP and continuation of medications. 
There are several limitations to this study. Due to its retrospective nature, patients were not randomized to each treatment group. Although applying different matching methods like coarsened exact [23, 30, 31] and propensity score matching [28] that we used in our previous studies could reduce the bias due to confounding variables, we were unable to use them here due to relatively small sample size. Additionally, this study was conducted at a single tertiary academic center and cannot easily be generalized to other practices.

In summary, we found that Ahmed glaucoma valves with same session trabectome surgery significantly decreased the rate of a hypertensive phase, and was associated with a lower postoperative IOP and fewer glaucoma medications.

Acknowledgments: We acknowledge support from NIH CORE Grant P30 EY08098 to the Department of Ophthalmology, from the Eye and Ear Foundation of Pittsburgh, and from an unrestricted grant from Research to Prevent Blindness, New York, NY. 


\section{References}

1. Bettin P, Khaw PT (2012) Glaucoma Surgery. Karger Medical and Scientific Publishers

2. Tseng VL, Coleman AL, Chang MY, Caprioli J (2017) Aqueous shunts for glaucoma. Cochrane Database Syst Rev 7:CD004918

3. Pakravan M, Esfandiari H, Yazdani S, et al (2017) Mitomycin C-augmented trabeculectomy: subtenon injection versus soaked sponges: a randomised clinical trial. Br J Ophthalmol 101:1275-1280

4. Gedde SJ, Panarelli JF, Banitt MR, Lee RK (2013) Evidenced-based comparison of aqueous shunts. Curr Opin Ophthalmol 24:87-95

5. Ramulu PY, Corcoran KJ, Corcoran SL, Robin AL (2007) Utilization of various glaucoma surgeries and procedures in Medicare beneficiaries from 1995 to 2004. Ophthalmology 114:2265-2270

6. Desai MA, Gedde SJ, Feuer WJ, et al (2011) Practice preferences for glaucoma surgery: a survey of the american glaucoma society in 2008. Ophthalmic Surg Lasers Imaging 42: . doi: 10.3928/15428877-20110224-94

7. Budenz DL, Barton K, Feuer WJ, et al (2011) Treatment outcomes in the Ahmed Baerveldt Comparison Study after 1 year of follow-up. Ophthalmology 118:443-452

8. Riva I, Roberti G, Katsanos A, et al (2017) A Review of the Ahmed Glaucoma Valve Implant and Comparison with Other Surgical Operations. Adv Ther 34:834-847

9. Esfandiari H, Hassanpour K, Knowlton P, et al (2017) Trabectome Surgery Combined with Baerveldt Glaucoma Implantation Negates Tube Fenestration

10. Nouri-Mahdavi K, Caprioli J (2003) Evaluation of the hypertensive phase after insertion of the Ahmed Glaucoma Valve. Am J Ophthalmol 136:1001-1008

11. Ayyala RS, Zurakowski D, Smith JA, et al (1998) A clinical study of the Ahmed glaucoma valve implant in advanced glaucoma. Ophthalmology 105:1968-1976

12. Eibschitz-Tsimhoni M, Schertzer RM, Musch DC, Moroi SE (2005) Incidence and management of encapsulated cysts following Ahmed glaucoma valve insertion. J Glaucoma 14:276-279

13. Esfandiari H, Loewen NA, Hassanpour K, et al (2017) Fuchs Heterochromic Iridocyclitis-Associated Glaucoma: A Retrospective Comparison of Primary Ahmed Glaucoma Valve implantation and Trabeculectomy with MMC

14. Shelton L, Rada JS (2007) Effects of cyclic mechanical stretch on extracellular matrix synthesis by human scleral fibroblasts. Exp Eye Res 84:314-322

15. Tripathi RC, Li J, Chan WF, Tripathi BJ (1994) Aqueous humor in glaucomatous eyes contains an increased level of TGF-beta 2. Exp Eye Res 59:723-727

16. Pakravan M, Rad SS, Yazdani S, et al (2014) Effect of early treatment with aqueous suppressants on Ahmed glaucoma valve implantation outcomes. Ophthalmology 121:1693-1698

17. Yazdani S, Doozandeh A, Pakravan M, et al (2017) Adjunctive triamcinolone acetonide for Ahmed glaucoma valve implantation: a randomized clinical trial. Eur J Ophthalmol 27:411-416

18. Smith M, Geffen N, Alasbali T, et al (2010) Digital ocular massage for hypertensive phase after Ahmed valve surgery. J Glaucoma 19:11-14 
19. Francis BA, See RF, Rao NA, et al (2006) Ab Interno Trabeculectomy: Development of a Novel Device (Trabectome ${ }^{\mathrm{TM}}$ ) and Surgery for Open-Angle Glaucoma. J Glaucoma 15:68

20. Kaplowitz K, Bussel II, Honkanen R, et al (2016) Review and meta-analysis of ab-interno trabeculectomy outcomes. Br J Ophthalmol 100:594-600

21. Loewen RT, Roy P, Parikh HA, et al (2016) Impact of a Glaucoma Severity Index on Results of Trabectome Surgery: Larger Pressure Reduction in More Severe Glaucoma. PLoS One 11:e0151926

22. Gedde SJ, Schiffman JC, Feuer WJ, et al (2005) The tube versus trabeculectomy study: design and baseline characteristics of study patients. Am J Ophthalmol 140:275-287

23. Neiweem AE, Bussel II, Schuman JS, et al (2016) Glaucoma Surgery Calculator: Limited Additive Effect of Phacoemulsification on Intraocular Pressure in Ab Interno Trabeculectomy. PLoS One 11:e0153585

24. Won HJ, Sung KR (2016) Hypertensive Phase Following Silicone Plate Ahmed Glaucoma Valve Implantation. J Glaucoma 25:e313-7

25. Molteno A, Dempster AG, Mills KB (1988) Methods of controlling bleb fibrosis around draining implants. In: Glaucoma. Proc 4th Int Symposium of North Eye Institute. Manchester, UK. pp 192-211

26. Seah SKL, Gazzard G, Aung T (2003) Intermediate-term outcome of Baerveldt glaucoma implants in Asian eyes. Ophthalmology 110:888-894

27. Allan EJ, Khaimi MA, Jones JM, et al (2015) Long-term efficacy of the Baerveldt $250 \mathrm{~mm} 2$ compared with the Baerveldt $350 \mathrm{~mm} 2$ implant. Ophthalmology 122:486-493

28. Kostanyan T, Shazly T, Kaplowitz KB, et al (2017) Longer-term Baerveldt to Trabectome glaucoma surgery comparison using propensity score matching. Graefes Arch Clin Exp Ophthalmol. doi: 10.1007/s00417-017-3804-9

29. Mosaed - Clin Surg Ophthalmol S, 2014 (2014) The first decade of global trabectome outcomes. touchophthalmology.com

30. Parikh HA, Bussel II, Schuman JS, et al (2016) Coarsened Exact Matching of Phaco-Trabectome to Trabectome in Phakic Patients: Lack of Additional Pressure Reduction from Phacoemulsification. PLoS One 11:e0149384

31. Dang Y, Kaplowitz K, Parikh HA, et al (2016) Steroid-induced glaucoma treated with trabecular ablation in a matched comparison with primary open-angle glaucoma. Clin Experiment Ophthalmol 44:783-788 
Figures and Tables

Table 1

Table 1. Baseline patients characteristics.

\begin{tabular}{|c|c|c|c|c|c|}
\hline & & \multirow[b]{2}{*}{ Total } & \multicolumn{2}{|c|}{ Group } & \multirow[b]{2}{*}{$\mathbf{P}$} \\
\hline & & & AT & A & \\
\hline \multirow[t]{2}{*}{ Age } & Mean \pm SD & $67.1 \pm 15.4$ & $64.4 \pm 15.6$ & $69.3 \pm 15.1$ & $0.107 \dagger$ \\
\hline & Median (range) & 68.5 (25 to 95$)$ & 64 (25 to 95$)$ & 74 (31 to 94$)$ & \\
\hline \multirow[t]{2}{*}{ BCVA } & Mean \pm SD & $0.71 \pm 0.74$ & $0.56 \pm 0.56$ & $0.87 \pm 0.88$ & $0.057 \ddagger$ \\
\hline & Median (range) & 0.44 (0 to 3.3$)$ & 0.3 (0 to 2.8 ) & 0.51 (0 to 3.3 ) & \\
\hline \multirow[t]{2}{*}{ IOP } & Mean \pm SD & $27.4 \pm 10.3$ & $26.6 \pm 10.1$ & $28.8 \pm 10.2$ & $0.121 †$ \\
\hline & Median (range) & 26 (8 to 56$)$ & 23.5 (9 to 56 ) & 28 (8 to 56$)$ & \\
\hline \multirow[t]{2}{*}{ Medications } & Mean \pm SD & $2.6 \pm 1.3$ & $2.6 \pm 1.2$ & $2.6 \pm 1.4$ & $0.814 \ddagger$ \\
\hline & Median (range) & $3(0$ to 7$)$ & $3(0$ to 5$)$ & $3(0$ to 7$)$ & \\
\hline \multirow[t]{2}{*}{ Sex } & $\mathrm{F}$ & $60(56.1 \%)$ & 25 (52.1\%) & 35 (59.3\%) & $0.453^{*}$ \\
\hline & M & 47 (43.9\%) & $23(47.9 \%)$ & $24(40.7 \%)$ & \\
\hline \multirow[t]{2}{*}{ Phacoemulsification } & No & 72 (70.6\%) & $31(72.1 \%)$ & $41(69.5 \%)$ & $0.776^{*}$ \\
\hline & Yes & $30(29.4 \%)$ & $12(27.9 \%)$ & $18(30.5 \%)$ & \\
\hline \multirow[t]{4}{*}{ Type of glaucoma } & POAG & $80(74.8 \%)$ & $38(79.2 \%)$ & $42(71.2 \%)$ & $0.680 * *$ \\
\hline & CACG & $11(10 . .2 \%)$ & $4(8.3 \%)$ & $7(11.8 \%)$ & \\
\hline & PXF & $10(9.3 \%)$ & $4(6.8 \%)$ & $6(10.1 \%)$ & \\
\hline & Other & $6(5.7 \%)$ & $2(4.1 \%)$ & $4(6.7 \%)$ & \\
\hline
\end{tabular}

† Based on t-test., $\ddagger$ Based on Mann-Whitney test., * Based on Chi-Square test., ** Based on Fisher exact test. 


\section{Table 2}

Table 2. Comparison of postoperative changes in intraocular pressure between AT and A.

\begin{tabular}{|c|c|c|c|c|c|c|c|c|}
\hline \multirow[b]{2}{*}{ Time } & \multirow[b]{2}{*}{ Total } & \multicolumn{2}{|c|}{ Group } & \multirow[b]{2}{*}{ Diff } & \multicolumn{2}{|c|}{$95 \% \mathrm{Cl}$} & \multirow[b]{2}{*}{$\mathrm{Pt}$} & \multirow[b]{2}{*}{ VarDiff Pł } \\
\hline & & AT & A & & & & & \\
\hline Baseline & $27.4 \pm 10.3$ & $26.6 \pm 10.1$ & $28.8 \pm 10.2$ & 3.2 & -0.8 & 7.2 & 0.12 & 0.843 \\
\hline \multirow[t]{2}{*}{ Day 1} & $12.8 \pm 10.6$ & $11 \pm 6.9$ & $14.5 \pm 12.8$ & 3.5 & -0.7 & 7.7 & 0.09 & $<0.001$ \\
\hline & $-14.4 \pm 14.78$ & $-14.65 \pm 10.4$ & $-14.1 \pm 17.8$ & 0.5 & -5.5 & 6.4 & 0.87 & 0.001 \\
\hline \multirow[t]{2}{*}{ Day 7} & $12.1 \pm 7.6$ & $11 \pm 6.7$ & $13 \pm 8.3$ & 2 & -1.1 & 5 & 0.2 & 0.24 \\
\hline & $-15.5 \pm 11.78$ & $-14.61 \pm 9.1$ & $-16.2 \pm 13.6$ & -1.7 & -6.4 & 3.1 & 0.48 & $<0.001$ \\
\hline \multirow[t]{2}{*}{ Month 1} & $17.3 \pm 7.9$ & $16.4 \pm 7$ & $18 \pm 8.6$ & 1.7 & -1.6 & 4.9 & 0.3 & 0.26 \\
\hline & $-9.89 \pm 11.18$ & $-9.14 \pm 9.6$ & $-10 \pm 12.3$ & -1.4 & -5.9 & 3.2 & 0.54 & 0.25 \\
\hline \multirow[t]{2}{*}{ Month 3} & $15.4 \pm 6.6$ & $14.7 \pm 5.4$ & $16.1 \pm 7.5$ & 1.4 & -1.3 & 4.1 & 0.31 & 0.03 \\
\hline & $-12.64 \pm 11.92$ & $-11.02 \pm 11.4$ & $-14.1 \pm 12$ & -3.1 & -8.1 & 1.9 & 0.21 & 0.49 \\
\hline \multirow[t]{2}{*}{ Month 6} & $17.1 \pm 6.6$ & $15.9 \pm 4.8$ & $18.8 \pm 8.2$ & 2.9 & -0.2 & 6 & 0.06 & 0 \\
\hline & $-9.83 \pm 10.76$ & $-9.49 \pm 10.51$ & $-10.3 \pm 11.2$ & -0.8 & -6.1 & 4.4 & 0.75 & 0.55 \\
\hline \multirow[t]{2}{*}{ Month 12} & $15.7 \pm 4.3$ & $14.7 \pm 3.3$ & $16.7 \pm 4.9$ & 2 & 0.1 & 3.9 & 0.04 & 0.02 \\
\hline & $-11.75 \pm 9.53$ & $-10.49 \pm 8.8$ & $-12.9 \pm 10.1$ & -2.5 & -6.9 & 1.9 & 0.26 & 0.83 \\
\hline
\end{tabular}

Diff: Difference. VarDiff: Variation difference. $†$ Based on t-test. $\ddagger$ Based on Leven’s test 


\section{Table 3}

Table 3: Logistic regression analyses of baseline predictors of an ocular hypertensive phase with AGV Implantation.

\begin{tabular}{|l|l|l|}
\hline Variables & B coefficient & P-value \\
\hline Baseline IOP & 1.04 & 0.05 \\
\hline Age & 0.98 & 0.35 \\
\hline Gender & 2.09 & 0.13 \\
\hline Phaco combination & 0.56 & 0.32 \\
\hline
\end{tabular}

IOP: intraocular pressure. 


\section{Figure 1}

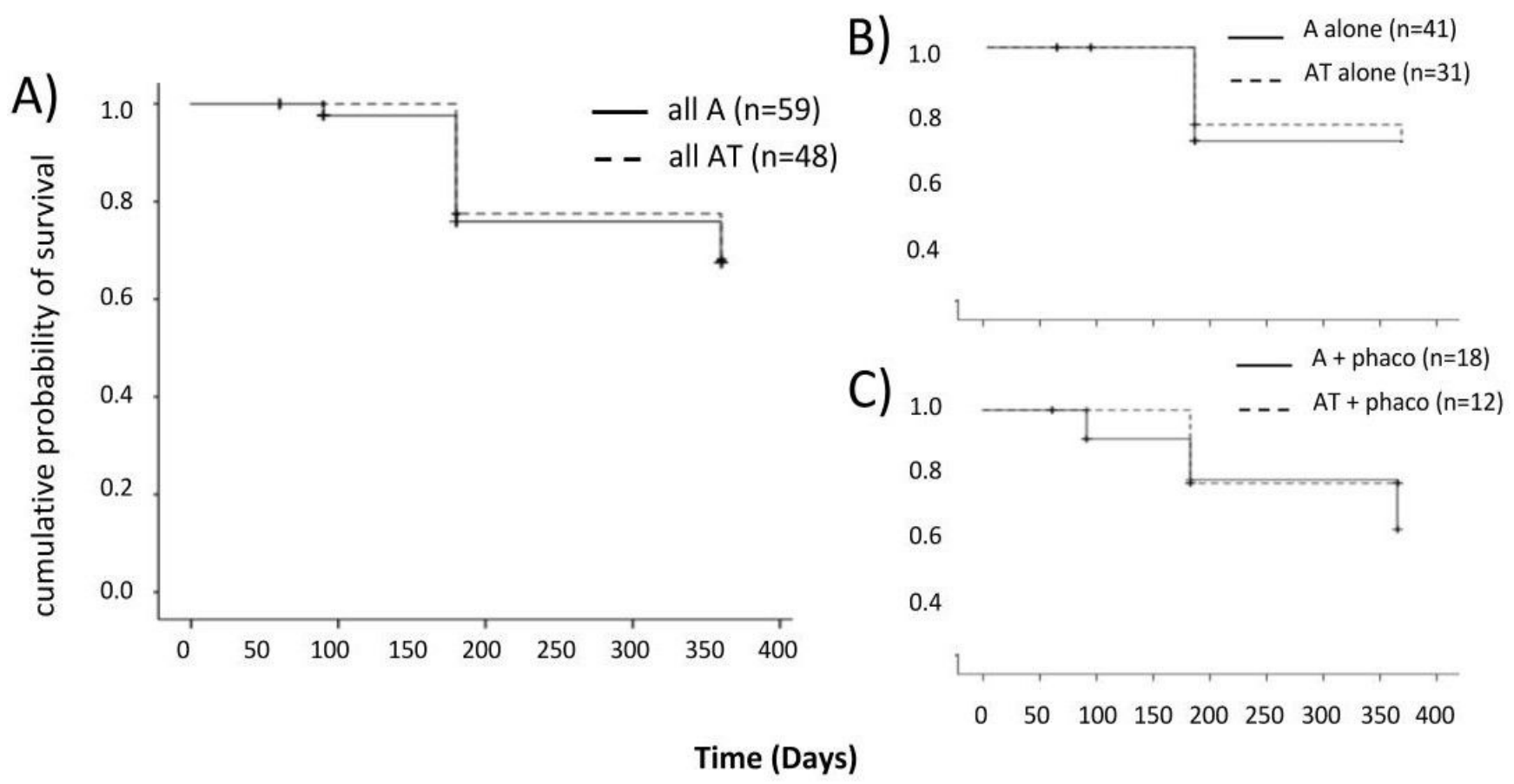

Figure 1: A) Kaplan-Meier survival plots for AT and A with success defined as a final IOP of $\leq 21 \mathrm{mmHg}$ and a $20 \%$ reduction from baseline. Success rates of AT and A was similar in both groups. B) survival plots of AT and A for subgroup analysis separated by B) glaucoma surgery alone (upper right) and C) same session phacoemulsification (lower right). 
Figure 2

Figure 2: IOP in AT and A. A) IOP in AT was significantly lower than A thorough postoperative follow-up visits (asterisks indicate statistically significant difference). B) AT + phaco had a lower IOP on day one compared to subsequent IOPs while IOP was higher on day one in A+phaco. C) AT and AT as standalone procedures.

Mean $\pm 95 \%$ confidence interval. 